\title{
A Study of Required English Skills for Healthcare Personnel in Bangkok-Based International Hospitals
}

\author{
*Inthira Pandey, Kantatip Sinhaneti \\ Shinawatra University Bangkok, Thailand \\ *in10247@hotmail.com
}

\begin{abstract}
The effective use of English skills is required for the Thai healthcare personnel. This study therefore aims to explore: 1) the use of the four English language skills by Thai healthcare personnel, 2) the minimum framework of effective interaction involvement for their situational contexts, and 3) the problems encountered when they communicate with foreign patients. This study revealed the following findings. Firstly, English speaking and listening skills were the two most important skills especially needed for healthcare personnel in their workplace. Secondly, grammatical errors and varieties of accents and pronunciation were seen as major communication problems. Lastly, the culture, social etiquettes, and values between healthcare personnel and foreign patients are required to build an internationally accepted reputation and meet patients' social, cultural, and linguistic needs. This study indicates that intensive practice of the language skills is needed for healthcare personnel in preparing Thailand for the medical hub, AEC and the global market.
\end{abstract}

Keywords: Healthcare Personnel, Communicative Skills, Language Skills, Interaction Involvement, and Problems Encountered

\section{Introduction}

Rationale for the Study: As global communication increases, English continues to become the first choice for cross-border communication worldwide (Kruekaew, 2008). The role of English in the workplace has been shaped dramatically by aspects of globalization as well as ASEAN Economic Community (AEC). English language is often seen as the key to gain access to global markets by non-English speaking companies. Likewise, employees who have the abilities to solve problems, communicate effectively with others (from the same culture and also from other cultures), work in teams, use online technologies effectively, and engage in critical analysis play an important role in shaping the organization's image. Effective communication and interpersonal skills are essential components for healthcare personnel in delivering good quality of healthcare services. Theorists and researchers (Putsch, 1985; Eggly, 2002; Planetree, 2008; Chapman, 2009) agreed that communicating effectively with patients is a cornerstone of providing quality healthcare. Whereas poor communication has potential to affect patient trust, understanding, diagnosis, and the treatment plan (Lee, 1997, quoted in Haynes, n.d). Moreover, patients who do not speak the same language as their healthcare providers consistently report lower satisfaction than those who share the same language as their providers (Bowen, 2001).The ability to communicate well belongs to the most important knowledge of each healthcare personnel which is an irreplaceable part of healthcare profession. This would only be possible if there is a proper communication. Cynthia (2001) suggested an experienced healthcare personnel should be aware of the patients' needs and make them more comfortable not only performs the given job. The interaction that occurs between healthcare personnel and patients is the foundation of healthcare settings since they contribute to the quality of healthcare. The U.S. department of health and humanity services (2001) stated that healthcare personnel who can communicate with patients with different social values, different languages, different views, and different religions can overcome healthcare barriers. In order to ensure patients' satisfaction, healthcare personnel should be aware and recognize foreign patient's cultural background and attitudes when communicating to avoid any necessary stereotyping and exceeds their expectations. To expand Thailand as a "medical hub", Koh-Shun (2004) pointed out that hospitals must overcome healthcare personnel language and cultural gaps by making them aware of the benefits of effective communication and understand patients who come from different cultures. Drawing upon prior research, this study presents a scope incorporated with ways in which communication takes place, the processes 
underlying health communication, and the barriers to communication in healthcare settings. Moreover, this study yields practice and policy implications to the government in preparing Thailand for a medical hub, AEC and the global market thus allows healthcare personnel to be more competitive when working locally, regionally or globally.

Purposes of the Study: The purposes of this study are:

- To explore the required English skills needed for healthcare personnel in Bangkok-based international hospitals in performing their job,

- To explore major problems they encounter when using English language in their workplace,

- To explore minimum framework of effective interaction involvement when the two parties did not share a native language

\section{Methodology}

Population and subjects: The population of this research study was fifty healthcare personnel in two Bangkok-based international hospitals namely, Bumrungrad International Hospital and Piyavate Hospital. Since Bangkok is the capital city of Thailand and considered as the first stop for medical tourists arriving from different parts of the world to seek medical treatment, the researcher foresees the increasing demand of the medical tourists which result choosing Bangkok-based international hospitals to conduct this study. Moreover, due to limited time constraint the researcher considered Bangkok-based international hospitals as the most suitable and accessible place to conduct this study. These two hospitals were selected based on their international reputation and hospital's image in terms of service, accommodation, facilities and equipment. Healthcare personnel in the selected hospitals serve as a liaison for foreign patients and families so their responsibilities suite the purpose of this study. In addition, they communicate with diverse patients and families through the use of oral and written mode of communication to meet patients' needs. The subjects in this study were fifty healthcare personnel who were randomly selected by the customer service manager based on their communicative abilities. Twenty-five healthcare personnel from each of these two hospitals received a questionnaire and five healthcare personnel from the twenty-five were interviewed randomly. The ten healthcare personnel were randomly selected based on their age, work experience, and language skills with the assistance and coordination from customer service manager.

Design of the study: This was a survey design. The methods employed in this study were mixed method: questionnaire for quantitative data and a structured interview for qualitative data. Below were the details of the instrument.

Questionnaire: There were two sets of pre-test questionnaire and final questionnaire. Questionnaires were used as an instrument to gather data about the needs of English skills for healthcare personnel in the two selected hospitals. Questionnaires were determined to be the best means of investigating this study because they require minimal time from the respondents and provide a flexible and convenient way to participate in the study. The questionnaires were constructed and developed based on a variety of previous related research, books, and journals concerning needs analysis functions and responsibilities as well as perspectives toward cultural needs. The questionnaires fell into five parts. The first part consisted of respondent's biographical data. The second part helped sought information about which of the four English language skills were emphasized in their work place to compare and contrast their usage in the hospital when interacting with foreign patients. The third part sought information about the degree of the importance of each of the four English language skills (listening, speaking, reading, and writing) perceived by the respondents in their job-related activities. The fourth part sought information about the respondent's feelings when communicating with foreign patients that may be relevant when they attempt to make themselves understood by foreign patients. All the feelings indicated the extent to which respondents generally feel after trying to make themselves understood by foreign patients. The final part sought information about the respondent's interaction involvement needs that may be relevant when they attempt to communicate with foreign patients. Each item describes respondent behavior when communicating with foreign patients. Finally, the questionnaires were distributed to fifty healthcare personnel in two Bangkok-based international hospitals. 
Structured interview: The quantitative analysis of the questionnaire was complemented by the qualitative data received from structured interviews to strengthen the design and clarify quantitative evaluation findings. In this study, a total of ten healthcare personnel from two Bangkok-based international hospitals were randomly selected to conduct the interview based on their age and number of years in healthcare services. They were interviewed face to face in Thai which was note taking by the researcher. The interview time varied from 15 - 20 minutes. It was necessary to ask permission from some respondents for a follow up interview, as more information might be required.

Data collection and analysis: For data collection, the questionnaire was developed and piloted with ten healthcare personnel to obtain prerequisite data relevant to the design of the final questionnaires. The data were collected during weekdays in December 2010. The ten healthcare personnel were asked to answer the questions and comments on the questionnaires' content. They were also asked to express their opinions concerning the current use of English language as well as the needs of English language skills. The questionnaires were revised on the basis of information obtained and the final questionnaires were developed. The reliability test in this research showed that the internal consistency was managed. The questionnaire had its reliability tested by means of the Cronbach's Alpha, which was .826, indicating high reliability. After the completion of the pilot study, all-necessary modifications were made in order to meet the requirements of a reasonable and sound questionnaire design. Data were collected from the following Bangkok-based international hospitals: Bumrungrad International Hospital and Piyavate Hospital. With respect to that, the questionnaires were distributed to twenty five healthcare personnel in each hospital on February $10^{\text {th }}, 2011$ and were collected in the following week. The researcher received fifty completed questionnaires on February $17^{\text {th }}, 2011$. For data analysis, SPSS was used to analyze the data from the final questionnaire collection. It is summarized and explained in terms of frequency and descriptive analysis. The statistical devices which are employed in this study include frequency analysis, descriptive statistics and the Cronbach's Alpha method is employed to ascertain the reliability of the response for those items which used a five point Likert scale. Additionally, the interview results were reviewed and manually recorded on Microsoft Excel Spreadsheet which was later summarized by the researcher.

\section{Results}

The results of the study were presented based on the three specific research questions of the study respectively. For the first research question: What are the needs of the four English language skills perceived by the healthcare personnel in Bangkok-based international hospitals? The results revealed that the majority of the respondents considered speaking and listening skills as most important skills needed to perform their jobs as shown in the figure below.

Figure 1: English skills required by healthcare personnel

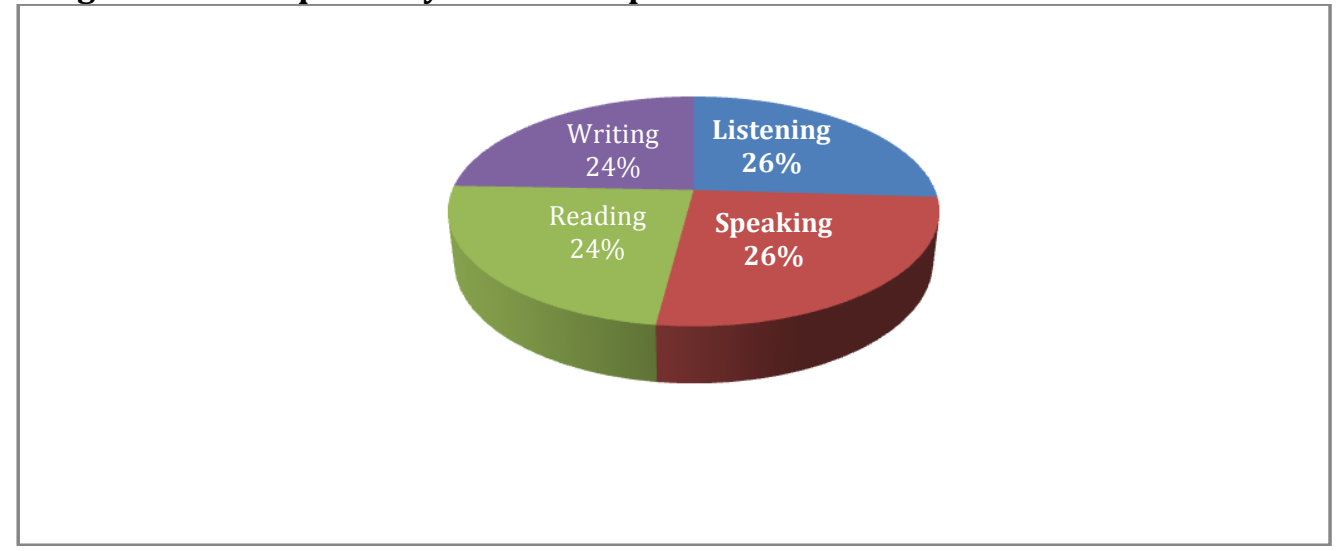

The above figure showed that the responses of the healthcare personnel on the four language skills needed for their job are not considerably different: speaking and listening $26 \%$, reading and writing $24 \%$ respectively. In details, $26 \%$ of the respondents considered speaking and listening skills as most important skill while $24 \%$ of the respondents considered reading and writing skills as important skill. Therefore, it can 
be measured that effective communications between healthcare personnel and foreign patients is crucial and the relationship must be managed effectively for successful performances. English language is used widely and thus plays an important role in their present job. Healthcare personnel foresee the needs to communicate effectively with foreign patients with varieties of English spoken, which enable them to convey the intended message correctly, thus result in patient's satisfaction. Speaking is the valuable skill in the oral mode which involves more than just pronouncing words. Therefore, it is the respondents' ability to explain and present their ideas in clear English to diverse patients and meet their needs. In healthcare settings, there are certain work solutions when speaking skill is necessary such as solving patient's problem, asking for relevant information, relating feeling, etc. Moreover, healthcare personnel are required to have active listening skillsto ensure a successful interaction through techniques that facilitate discussion, demonstrating that they truly listen and understand the information provided by foreign patients and respond back appropriately to avoid any miscommunication. Thus, effective listening skills help acquire better information, save time, solve problems, and reduce errors. Alternatively, poor listening creates misunderstandings, wastes time, and allows for mistakes.

The interview results supported those of the questionnaire results since the respondents were required to interact with foreign patients on their daily basis. Moreover, they are required to actively listen (in order to understand the patient's message) and provide appropriate responses in (verbal communication) with a professional attitude, showing professional courtesy at all times. This will enable them to deliver a quality healthcare service resulting in patient's satisfaction hence create a positive image for the hospital. The interviews were summarized as follow:

"The first group is confident with their speaking skills because they have to communicate with diverse patients in order to deliver healthcare service to satisfy patients' needs, hence building a good relationship."

"The second group is confident with their listening skills because they have to pay full attention to listen to patients in order to comprehend their request and provide an appropriate feedback which allows them to confidently build trust with patients."

Since English is used as a mean of communication in Bangkok-based international hospitals, all healthcare personnel are required to have effective communication skillsto interact with foreign patients. Effective speaking skills help create clearer communication and allow less chance of misunderstandings. While effective listening skills help acquire better information, save time, solve problems, and reduce errors. Therefore, it can be concluded that healthcare personnel's English skills plays an important role not only to themselves but to the hospital's image. For the second research question: What are the major problems encountered by healthcare personnel in Bangkok-based international hospitals in using English language in the workplace? The result revealed that grammatical errors and varieties of accents and pronunciation were seen as major communication problems which needed to be improved.

Figure 2: Problems encountered by healthcare personnel

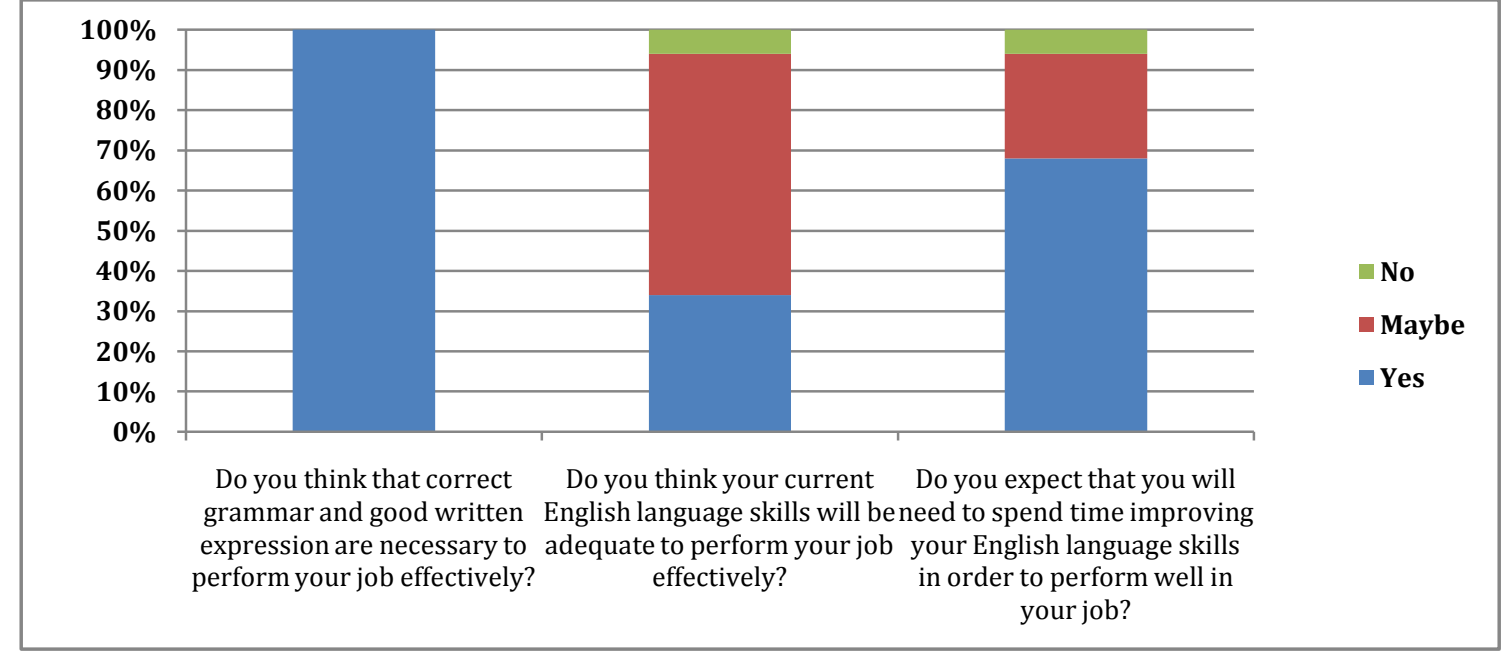


The results revealed that it was agreed by all respondents that grammar and good written expression are necessary for their job effectively. It is considerately agreed by all respondents $100.0 \%$ that correct grammar and good written expression are necessary to perform their job effectively. However, $60.0 \%$ of the respondents think that their current English language skills maybe adequate to perform their job. As a result, $68.0 \%$ of the respondents expected that they will need to spend time improving their English language skills in order to perform well in their job. It can be concluded that not all of their English language skills are effective and can serve them to achieve their goals. The respondents agreed that grammatical errors may affect their job performance because either success or failure may depend upon it. Therefore, it's necessary for them to be aware of the effectiveness of correct grammar and good written expression because either success or failure may depend upon it. The interviews were summarized as follow:

"The respondents considered grammatical errors as problems which restricted their monthly report writing. In addition, varieties of accents and pronunciation used by diverse patients were viewed as communication barrier".

The interview results supported those of the questionnaire results since the language and cultural differences often underlie communication problems in healthcare settings. The respondents recognized effective communication as a priority across the healthcare context because it directly affects the quality of patient care, safety, medical outcomes and patient satisfaction. Furthermore, grammatical errors and good written expressions, varieties of accents, and pronunciation of diverse patients were viewed as problems encountered across healthcare settings. Therefore, the respondents considered acquiring appropriate usage of English language emphasizing on varieties of accents and pronunciation which would facilitate them to deliver a quality health service. In order to keep track of the work progress, the respondents required more trainings and team meetings to enable them share their experiences explicitly when communicating with foreign patients. In details, based on the interviews conducted with healthcare personnel in Bangkok-based international hospitals, the respondents' have difficulties in pronunciation which were consistent with the respondents' awareness of pronunciation and speech organ difficulties include the following errors: words with / $\mathrm{r} /$, usually pronounced as /l/; words with /ei/, usually pronounced as /e/; words with /z/, usually pronounced as /s/or voiceless; words with /f/, usually pronounced as /p/; words with /v/, usually pronounced as $/ \mathrm{f} /, / \mathrm{p} /$, and $/ \mathrm{w} /$; words with $/ æ /$, usually pronounced as /a:/; words with /eI/, usually pronounced as /e/; words with /əv/, usually pronounced as /p/; words with /d/ and /s/, usually pronounced as $/ \mathrm{t} /$; words with $/ \mathrm{g} /$, usually pronounced as $/ \mathrm{k} /$; words with $/ \theta /$, usually pronounced as $/ \mathrm{s} /$; words with $/ \mathrm{J} /, / \mathrm{t} /$ and $/ \mathrm{d} /$ /, usually pronounced as $/ \mathrm{t} /$ or $/ \mathrm{t} /$; on the other hand, words with $/ \mathrm{t} /$, usually pronounced as $/ \mathrm{n} /$.

It is obvious that language barriers continue to lower the quality of care within the healthcare context. Thus, a commitment to ongoing professional development throughout their career path may be beneficial for them and help them overcome problems. However, regular team meetings can be conducted where a team of healthcare personnel share each other's core-skill reflecting on communication development. Team meetings are powerful weapons that allow healthcare personnel to highlight good and bad communication, or difficult situations encountered hence fosters and strengthens good working relationship. The interviews were summarized as follow:

"The respondents stated that more training and meeting should be urged among healthcare personnel to share their experiences. Moreover, knowledge form experts both linguistically and culturally can develop one's awareness related to their job".

For the third research question: What are the minimum frameworks of effective interaction involvement for healthcare personnel in Bangkok-based international hospitals when the two parties did not share a native language? In today's healthcare settings, it is fundamental for healthcare personnel to build rapport with patients and focus on them when addressing. Moreover, healthcare personnel must recognize that patients from diverse background may have different communication needs. Therefore, it's necessary to keep patients' expectations realistic and make sure patients know what you do. The result revealed that they ask for confirmation of understanding throughout the communication process to seek clarification and observe nonverbal clues for a shared understanding and develop alternate perspectives between themselves and foreign patients to build a good reputation and meet patients' social, cultural, and linguistic needs. 
Figure 3: Minimum framework of effective interaction involvement

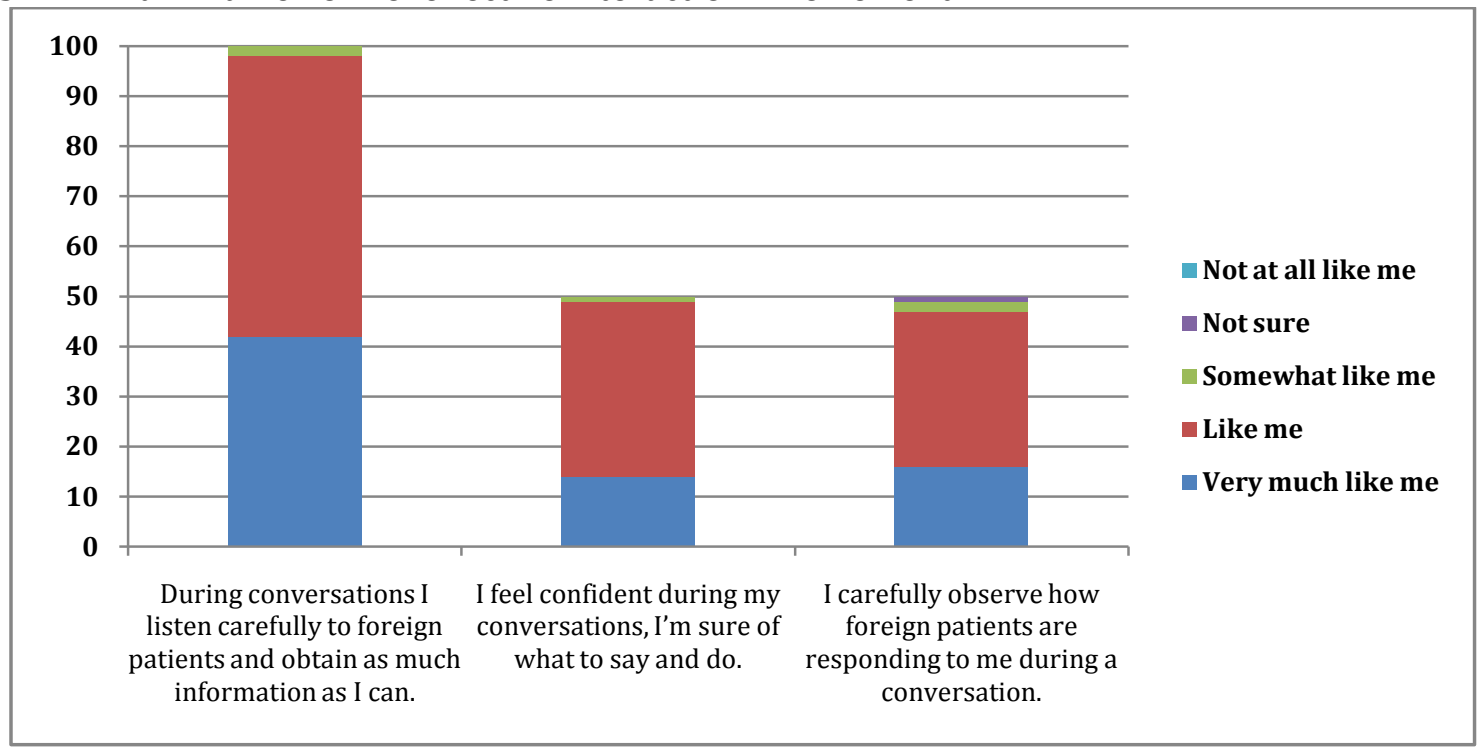

The above figure indicated how respondents communicate and describe respondent's behavior when communicating with foreign patients. $42 \%$ of the respondents tend to listen carefully to foreign patients and obtain as much information as they can. In addition, 32\% of the respondents feel confident during their conversations. However, $28 \%$ of the respondents carefully observe how foreign patients response to them during a conversation. It can be concluded that effective communication is an essential part of building and maintaining good relationships between healthcare personnel and foreign patients. The results revealed that healthcare personnel make themselves aware of the communication expectations of the for eign patients, yet accept the point that misunderstanding may occur during the communication process. Healthcare personnel have a responsibility to overcome the cause of the misunderstanding by familiarizing the needs, cultural expectations and language level of the foreign patients. They tendto ask for confirmation of understanding throughout the communication process to determine their own level of understanding and thus achieve effective communication. They generally feel happy, respected, and secure when they try to make themselves understood by foreign patients in the communication process. When communicating with foreign patients, healthcare personnel tendto seek clarification, and observe how foreign patients are responding throughout the communication process to determine their own level of understanding and thus achieve effective communication. Communication however often includes non-verbal clues such as tone of voice, facial expression, gestures, and body posture. Besides communication, healthcare personnel requires a shared understanding from the perspectives of both parties to understand and learn from each other, develop alternate perspectives, and thus meet each other's needs which is called effective interaction involvement. As a result, healthcare personnel feel confident when they tend to explain and present their ideas in clear English to diverse patients. The interviews were summarized as follow:

"The respondents recognized the needs of interaction involvement with patients such as eye contact, facial expression, and use of body language".

The interview results lend support to those of the questionnaire results since civilizations have brought in diversity in people's life from different parts of the world. Being able to communicate well is an essential skill which helps strengthen trust in the quality of medical care received and increases the likelihood of appropriate follow-up. Healthcare personnel anticipated the needs to understand the similarities and the differences between themselves and foreign patients in terms of culture, social etiquettes, and values in order to build a good reputation while staying thorough, focused, organized, and efficient. Language and cultural differences often underlie communication problems in healthcare settings. Culture differences are important to healthcare personnel in performing their job because people from different cultural backgrounds do not necessarily share the same knowledge or expectations about healthcare services. Thus, needs and wants of foreign patients' vary since they come from different countries with different background where diverse culture is associated. The interviews were summarized as follow: 
"The respondents pointed that the Dos and Don'ts of all cultures should be emphasized because one thing may have different meanings or used different in the patient's culture/country."

\section{Conclusion}

Based on the results of the study, it can be concluded that, firstly, healthcare personnel required English speaking and listening skills to be two most important skills needed in solving patient's problem, relating feelings, building and maintaining good relationships which help acquired better information, save time, reduce communication errors, and thus meet each other's needs. Secondly, correct grammar, good written expression, varieties of accents and pronunciationwere regarded as problems encountered. Thus, a commitment to ongoing professional development throughout their career path may be beneficial for them and help them overcome problems. Lastly, healthcare personnel foresee the needs to understand the similarities and the differences between themselves and foreign patients in terms of culture, social etiquettes, and values in order to build a good reputation while staying thorough, focused, organized, and efficient.

Discussion: The results of this study lend supports to previous research study at least in three main points. Firstly, on required English skills, the results of the study supported with the following prior studies. For example, Brown (1994) pointed that speaking and listening skills were most often used skills in an English language context. In accordance to Brown's perspective, Alharby (2005) stated that listening and speaking skills were perceived as most important skills in the careers of health professionals. Though, Tasci (2007) found out that speaking skills were highly important skill needed for medical students in an EFL context. On the other hand, Taylor and Campbell (1999) foresee communication as a two-way process that requires clarification and reinforcement, and should be reflected back by repetition to confirm understanding. Secondly, on problems encountered, the result of this study lends support to the following prior studies. For instance, Albostan (2012) found out that pronunciation, writing and grammar are slightly more problematic skills then reading, speaking, listening and vocabulary. Similarly, Gass (2012) revealed that lack of vocabulary, pronunciation problems, accents, and rate of speech were perceived as problems for Thai nurses. On the other hand, Campbell (1999) agreed that opportunities should be provided to debrief staff after difficult events. Furthermore, Livesley (2000) suggested that team meetings should be undertaken to discuss problems, appraise staff, generate new ideas, and introduce changes to avoid poor communication. Alternatively, Chapman (2009) aimed to improve communication in healthcare setting by establishing a patient-centered healing environment in which open and transparent communication is the norm. To sum up the problems encountered, Heitner (2011) addressed employers in raising employees' awareness of language needs by offering language studies to fill the language skills gap.

Finally, on the minimum frameworks of effective interaction involvement, the results of this study were consistent with the following prior studies. To illustrate, Betancourt, Green, Emilio Carrillo (2002) described the ultimate goal in healthcare settings is to deliver the highest quality of care to every patient, regardless of race, ethnicity, cultural background, or English proficiency. Additionally, Sysoyev (2003) fostered the abilities to understand the characteristics of one's culture based on ethnicity, gender, social class, location, sexual orientation, language, etc. Significantly, Katterl (2010) viewed warm greeting, eye contact, a brief nonmedical interaction, or checking on an important life event as a way to build rapport relationship between healthcare providers and patients. Stemming from the above studies, Charoensook (n.d) revealed that cultural differences affect expectations and behaviors of individuals seeking medical care.It is widely accepted that the ability to communicate effectively, clearly, and appropriately is essential for healthcare personnel to cope with patient's needs. Effective communication requires a cluster of particular skills, behaviors and approaches, supported by appropriate attitudes and values.Patients, on the other hand, can experience measurable health benefits when healthcare personnel not only use effective communication skills but rather provide a good environment, give accurate information, and encourage positive motivation to patients. It is important for healthcare personnel to take into account where their attitudes originate when working with cross-cultural patients. With this understanding, healthcare personnel can then start to adapt their own behavior hence gain acceptance by communicating with foreign patients more on their terms. Cross-cultural communication is difficult, yet it is essential and rewarding. To be successful with this skill, healthcare personnel must try to adjust and learn from mistakes. Nevertheless, be sensitive to each foreign patient as an 
individual, not just as part of a group. After all healthcare personnel should treat foreign patients as best as they can.

Implications: For pedagogical implications, this study indicates that intensive practice of the language skills is needed for healthcare personnel in preparing Thailand for the medical hub, AEC and the global market. Since English is used widely as the means to communicate, negotiate and execute transactions where one can be a native speaker of English or none are native speakers of English. It cannot be denied that the English language is a passport to success for a distinct nation state operating in the globalized marketplace. English is not only a tool to gain access to modern technology but also a key to professional accomplishment because international healthcare settings demands communication with foreign patients. There will be high mobility in the cross border labor market where English is expected to be an international language used in communication. As a result, those who do not have good English proficiency as a prerequisite skill may experience limited opportunities for employment because English-speaking abilities are preferred, particularly by international companies.

Suggestion for Further Study: Future research may replicate this research by using an interview or a focus group with native speaker of English to reflect their communication experiences and actively seek solutions to overcome communication and/or cultural barriers. Moreover, future research may be further conducted using interview or focus group with board of directors, executives, managers or supervisors to find out their opinion or point of view towards the healthcare personnel's English skills responding to the foregoing roadmap of AEC.Future research may replicate this research by conducting a research amongst all international hospitals in Thailand by using more respondents to identify the required English skills for healthcare personnel. Thus, identifying effective communication capabilities and actively seek solutions to overcome communication barriers.

\section{References}

Albostan, Y. (2012). An Investigation of the Occupational Language Needs of Diplomats Whose Second Language is English. Master's Thesis, Teaching English as a Foreign Language. Bilkent University, Ankara.

Alharby, M. (2005). ESP Target Situation Needs Analysis: The English Language Communicative Needs as perceived by Health Professionals in the Riyadh Area. Retrieved February 03, 2012 from http://faculty.ksu.edu.sa/majed/Documents/alharby_majid_200505_phd.pdf

Arakelian, C., Bartram, M. \& Magnall, A. (2003). Hospital English. United Kingdom: The Radcliffe Medical Press.

Betancourt, J. R., \& Green, A. R. \& Emilio Carrillo, J. (2002). Cultural Competence in Health Care: Emerging Frameworks and Practical Approaches. Retrieved September 19, 2012 from www.commonwealthfund.org/usr.../betancourt_culturalcompetence_576.pdf

Brown, H. D. (1994). Teaching by principles: an interactive approach to language pedagogy. Englewood Cliffs, NJ: Prentice Hall Regents.

Chapman, K. B. (2009). Improving Communication among Nurses, Patients, and Physicians. American Journal of Nursing, 109(11), 21-25.

Chareonsook, D. (n.d). Thai Doctor and Foreign Patients Communication during the Medical Care: Case Study in Thailand. Research Paper, Faculty of Communication Arts. Rangsit University, Thailand.

Chunlaka, P. (2010). International Patients' Satisfaction towards Nurses Service Quality at Samitivej Srinakar in Hospital. Master's Project, M.A. (Business English for International Communication). Bangkok: Graduate School, Srinakharinwirot University.

Cynthia, S. (2001). Importance of Communication in Nursing Care. Retrieved January 20, 2010 from http://laico.org/v2020resource/files/commninnursing_jul-sep01.pdf

Eggly, S. (2002). An ESP Program for International Medical Graduates in Residency. In T. Orr (Ed.), English for specific purposes (pp. 105-115). Alexandria, VA: TESOL.

Gass, J. (2012). Needs Analysis and Situational Analysis: Designing an ESP Curriculum for Thai Nurses. English for Specific Purposes World, ISSN 1682-3257, http://www.esp-world.info, Issue 36, vol. 12, 2012 
Haynes, K. M. (n.d). Do You Get Me? Exploring Cross-Cultural Communication between Refugees and Health Practitioners. Retrieved March 20, 2013 from http://web3.unt.edu/honors/eaglefeather/wpcontent/2010/08/Haynes-Kayla-Final.pdf

Heitner, K. L. (2011). Current and Future Language Demands in the Workplace: Proficiencies and Gaps. $\begin{array}{llll}\text { Retrieved January } & 25, & & \text { from }\end{array}$ http://apolloresearchinstitute.com/sites/default/files/language_vertical_report_final.pdf

Katterl, S. (2010). Doctor Patient Communication in Health Care Service Delivery: A Case of Tribhuvan University Teaching Hospital, Kathmandu. Master's Thesis. Master in Public Policy and Governance Program Department of General and Continuing Education. North South University, Bangladesh

Koh-shun, J. L. (2004). Healthcare Marketing in Thailand. Retrieved on December 27, 2011 from http://www.amchamthailand.com/asp/view_doc.asp?DocCID=1074

Kruekaew, T. (2008). Factors Affecting English Vocabulary Skill of Undergraduate Students at Prince of Songkla University, Pattani Campus. Master's Thesis. Master of Science in Research Methodology. Prince of Songkla University, Thailand.

Livesley, M. (2000). Morale within operating departments. British Journal of Theatre Nursing.

Planetree, S. F. (2008). Practical Approaches for Building a Patient-Centered Culture. Retrieved March 20, 2013 from www.planetree.org

Putsch, R. W. (1985). Cross-cultural Communication. The Special Case of Interpreters in Health Care $\begin{array}{llll}\text { Retrieved } \quad \text { March } & 2013 \text { from }\end{array}$ http://www.nyu.edu/dental/studentaffairs/diversitycourse/CrossCulturalCommunicationInterpret ers.pdf

Sysoyev, P. V. (2003). Integrating Language and Content. Retrieved February 24, 2013 from http://www.tesolmedia.com/articles/CC_Live_Differences.pdf

Tasci, C. (2007). An Analysis of Medical Students' English language needs. Unpublished Master's Thesis. Bilkent University, Ankara.

Taylor, M. \& Campbell, C. (1999). Organizational skills and tools. British Journal of Theatre Nursing.

U.S. Department of Health and Human Services, OPHS Office of Minority Health, (2001).National Standards for Culturally and Linguistically Appropriate Services in Health Care. Retrieved on March 17, 2011 from http://minorityhealth.hhs.gov/assets/pdf/checked/executive.pdf 